\title{
Boron Doping of Hydrogenated Amorphous Silicon Prepared by rf-co-Sputtering
}

\author{
M.M. de Lima Jr. ${ }^{1}$, F.L. Freire Jr. ${ }^{2}$, and F.C. Marques ${ }^{1}$ \\ ${ }^{1}$ Instituto de Física "Gleb Wataghin", Universidade Estadual de Campinas \\ Unicamp, 13083-970 Campinas, SP, Brasil \\ ${ }^{2}$ Pontifícia Universidade Católica do Rio de Janeiro, \\ PUC-RJ, Rio de Janeiro - RJ, Brazil
}

Received on 23 April, 2001

\begin{abstract}
This paper addresses the doping mechanism of amorphous semiconductors through the investigation of boron doped $\mathrm{rf}$ co-sputtered amorphous hydrogenated silicon. The activation energy and room temperature conductivity varied from 0.9 to $0.3 \mathrm{eV}$ and from $10^{-12}$ to $10^{-4} \mathrm{Ohm}^{-1} . \mathrm{cm}^{-1}$, respectively, by ranging the boron concentration from 0 to 3 at.\%. These ranges of electronic properties are of the same order of those reported for samples prepared by plasma enhanced chemical vapor deposition (PECVD). In spite of these similarities, there are some relevant differences. The doping efficiency, at low boron concentrations, is much lower than that of PECVD samples. In addition, the creation of deep defects (dangling bonds) does not follow the square root power law dependence on the boron concentration as proposed by Street and observed in PECVD samples. These differences are associated with the density of defects, which is much higher in films prepared by sputtering. The increase in the deep defect density is more likely related to topological disorder, introduced by the presence of a high concentration of inactive impurities.
\end{abstract}

\section{Introduction}

The observation of doping in a-Si:H thin films, in 1975, by Spear and Le Comber [1] was an important breakthrough in the field of amorphous semiconductors, since at that time most people use to believe that disordered materials could not be doped at all. Indeed, due to the lack of constraints of an amorphous network, the 8-N Mott's rule [2] predicts that all the elements introduced into those materials would be bonded at their own configuration. Thus, the impurities could not act as donors or acceptors. A modified Mott's rule (including charged atoms) was then proposed by Street [3] in order to explain the mechanism that allows the experimentally observed doping of amorphous semiconductors. This model managed to explain the low and variable doping efficiency and the creation of additional dangling bonds in hydrogenated amorphous silicon and germanium prepared by plasma enhanced chemical vapor deposition (PECVD), doped either with boron or phosphorous. This model is supposed to be very general: independent of deposition technique, and valid for silicon and germanium doped with any element of columns III and V. Nevertheless, arsenic doped a-Si:H deposited by PECVD [4], as well as a-Ge:H prepared by rf-sputtering using column III and V dopants other than B and P [5], do not fit Street's model. This is an indication that other mechanism, besides charge injection, is also taking place. Though a lot of researches have been performed and several technological devices have been developed using doped amorphous silicon, the actual doping mechanism of amorphous semiconductors is, until now, not completely understood. In this work, boron doped a-Si:H films was studied in order to investigate the validity of Street's model in films prepared by rf-sputtering, which have much higher concentration of deep defects than films prepared by PECVD.

\section{Experimental details}

The samples were prepared in a Leybold Z400 rfsputtering system co-sputtering small pieces of boron on a 3" diameter silicon target. The boron to silicon area ratio (from 0 to $13 \%$ ) was the only parameter modified. All the other deposition parameters were kept fixed (bias of $1000 \mathrm{~V} ; 250{ }^{\circ} \mathrm{C}$ substrate temperature; $\mathrm{H} 2$ flux of $8 \mathrm{sccm}$; $\mathrm{Ar}+\mathrm{H} 2$ pressure of $8 \times 10^{-2}$ mbar; and $120 \mathrm{~min}$ of deposition time). The films, of about $0.6-0.8 \mu \mathrm{m}$ thick, were deposited onto crystalline silicon and quartz substrates, for the measurements of optical properties and the concentration of hydrogen and boron, and on 7059 corning glass covered with a thin layer of chromium, for conductivity 
measurements.

The actual concentration of boron in the films was determined using the nuclear reaction ${ }^{11} \mathrm{~B}(\mathrm{p}, \mathrm{a}) 2 \mathrm{a}[6]$. A quite linear correlation between the boron area in the target and the boron content in the film was observed. The concentration of bonded hydrogen was determined by infrared absorbance using a conventional Nicolet FTIR spectrometer and the well-known calibration constant for the hydrogen-silicon wagging vibration (at $640 \mathrm{~cm}^{-1}$ ) [7]. The absorption coefficient and pseudo-gap were determined by the procedure developed by Swanepoel [8] using the transmittance data on the visible region. Photothermal Deflection Spectroscopy (PDS) was used to obtain the density of dangling bonds and the Urbach energy. The conductivity measurements were performed using a sandwich setup (the top contacts were evaporated aluminum dots) in order to avoid surface effects, which are usually present in measurements performed using conventional parallel contacts. The measurements were taken in the 300-450 $\mathrm{K}$ temperature range at a rate of $3 \mathrm{~K} / \mathrm{min}$, during the cooling cycle. All samples were measured at the same, fixed, voltage and in the ohmic regime.

\section{Results}

Fig. 1 shows the results of the conductivity measurements as a function of the boron content. Fig. 1a displays the room temperature conductivity, while Fig. 1b shows the activation energy. For comparison, Fig. 1 also includes data from Spear and Le Comber [9] for doped a-Si:H prepared by PECVD using diborane as a source of boron; and by Kalbitzer et al. [10] for a-Si:H also prepared by PECVD, but introducing boron into the films by implantation. One can notice that in our samples we manage to change the room temperature conductivity, as well as the activation energy, in the same range obtained for PECVD films. The most important difference is the large amount of boron required, in the sputtered films, to achieve the same parameters obtained for glow discharge films.

Fig. 2 presents the variation of the defects density, inferred by PDS spectroscopy, as a function of the boron content. The dotted line, with a log-log slope of 0.65 , is a power law fit to the data. The films with boron content higher than $3 \times 10^{20}$ atoms $/ \mathrm{cm}^{3}$ were excluded from this fitting because at about this concentration the optical and structural properties start to change significantly. In particular, the hydrogen concentration and band gap decrease as the boron concentration increases in that range.

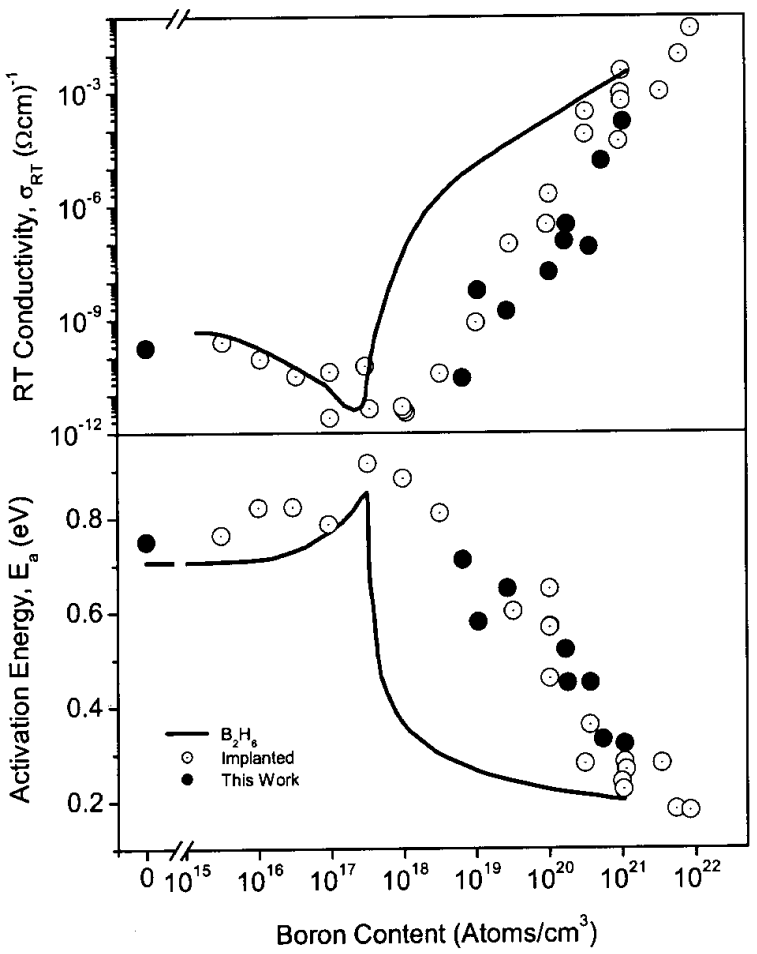

Figure 1. Activation energy and room temperature conductivity of boron doped a-Si:H as a function of the boron concentration. The full circles are co-sputtered samples; the open circles are boron implanted PECVD samples; and the solid line is the result obtained for PECVD films using diborane as a source of boron.

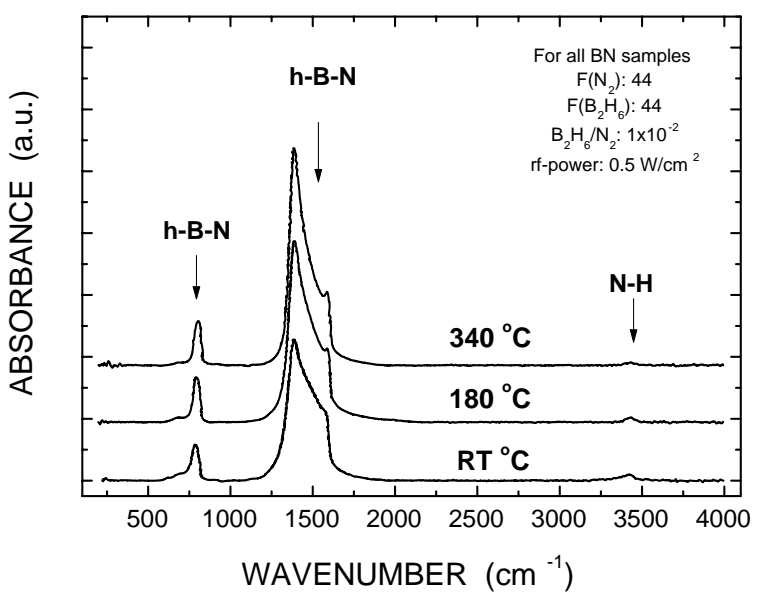

Figure 2. Deep defect density as a function of boron concentration. The solid line is a linear fit, whose slope is 0.65 .

Fig. 3 shows the relation between the Urbach energy and the density of dangling bonds. Data from literature, used by Stutzmann [11] to support his model of spontaneous conversion of weak bonds into dangling bonds, were also added to the figure for the sake of comparison and to increase the range of data for testing his model. 


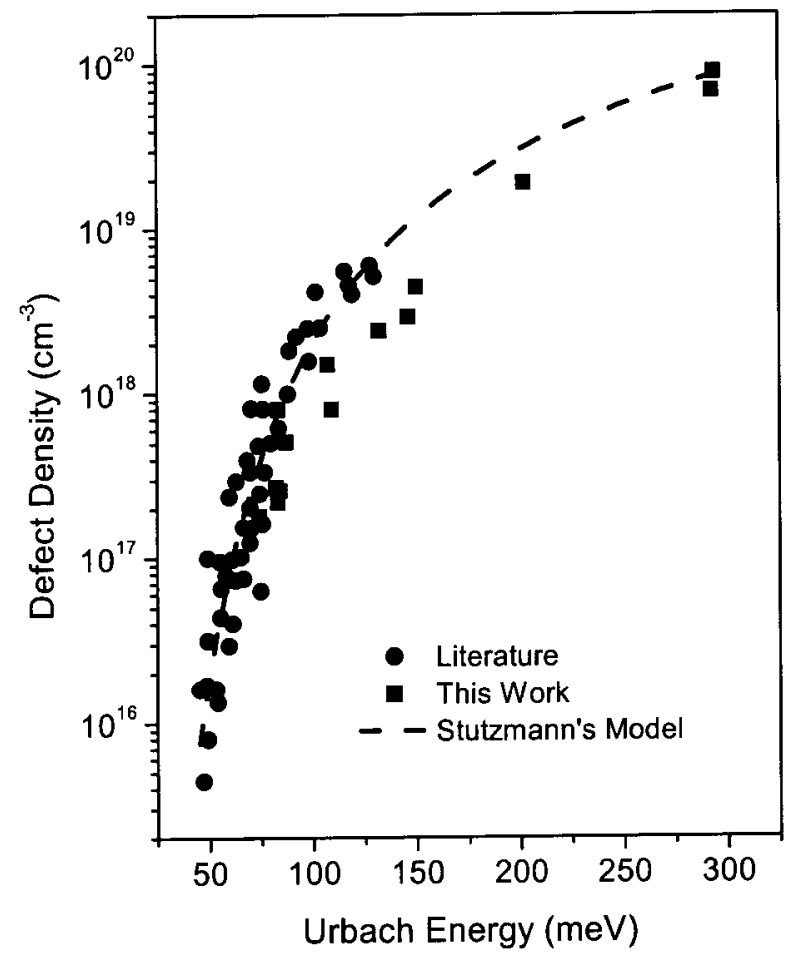

Figure 3. Deep defect density as a function of Urbach energy. The circles are data from literature and the squares are data from this work (co-sputtered samples). The dashed line is calculated according to the weak bond to dangling bond conversion model proposed by Stutzmann.

\section{Discussion}

The Street's model claims that the ratio between the four-fold and three-fold coordinated silicon atoms is given, on equilibrium, by the chemical reaction:

$$
\mathrm{Si}_{4}^{0}+\mathrm{X}_{3}^{0} \leftrightarrows \mathrm{Si}_{3}^{+}+\mathrm{X}_{4}^{-}
$$

where the subscripts are the coordination number and the superscripts are the charge state. $\mathrm{X}$ is any impurity from III and V periodic table columns. From this simple chemical reaction, using the mass action law, one can find the dangling bonds density $\left(\mathrm{N}_{D}\right)$, as well as the doping efficiency $(\eta)$, as a function of the impurity concentration. The dangling bond density varies directly with the square root of impurity content $\left(\mathrm{N}_{D}\right.$ $\left.\alpha \quad[\mathrm{X}]^{\frac{1}{2}}\right)$, while the doping efficiency is inversely proportional to the square root of the impurity content $(\eta$ $\left.\alpha[\mathrm{X}]^{-\frac{1}{2}}\right)$.

Fig. 2 shows that, in our samples, $\mathrm{N}_{D} \alpha[\mathrm{B}]^{0.65}$, i.e., it does not follow the square root power law function predicted by the Street's model. This disagreement must be related with the electronic structure differences between the sputtered and PECVD samples. First of all, the density of deep defects on the intrinsic sputtered material is almost 500 times higher than that of films deposited by PECVD. Besides, the Urbach energy, of about $80 \mathrm{meV}$, is also much higher than that obtained from good PECVD samples, which is about $50 \mathrm{meV}$ or lower. Indeed, as it is well know, a high density of deep defects must pin the Fermi level near the midgap. As a consequence one must incorporate much more boron in sputtered films than in PECVD ones in order to obtain the same activation energy variation. For instance, in Fig. 1 one can notice that in order to reach values of $\mathrm{Ea} \sim{ }^{\sim} 0,4 \mathrm{eV}$ and $\mathrm{C}_{R T}{ }^{\sim} 10^{-7} \mathrm{~W}^{-1} \mathrm{~cm}^{-1}$, the boron incorporation in the sputtered films is almost 3 orders of magnitude higher than that of PECVD films. Therefore, the effect of distortion around the impurity vicinity due to the incorporation of such a high concentration of atoms with coordination 3 cannot be neglected. In this process, the amount of $\mathrm{Si}-\mathrm{Si}$ weak bonds increases and a certain quantity of dangling bonds must be created. This statement is supported by the results obtained for the Urbach energy, since its relation with the dangling bonds density (Fig. 3) is quantitatively in good agreement with the model proposed by Stutzmann [11]. This model explains how an increase of weak bonds, expressed in terms of Urbach energy, can produce an excess of dangling bonds. Thus, the dangling bond production in boron doped amorphous silicon prepared by sputtering cannot be explained only in terms of charge injection Street's model as in PECVD samples. Other features such as topological disorder created by the large amount of boron introduced into the films must be taken into account.

\section{Conclusion}

In summary, we managed to dope amorphous silicon with boron, using a co-sputtering technique, with activation energy and conductivity of the same order of those obtained for PECVD films. However, the amount of boron required to shift the Fermi level is about 3 orders of magnitude higher than that necessary in PECVD samples. It was also observed that the chargeinduced model does not describe satisfactorily the production of dangling bonds. However, the data fits very well to the Stutzmann model based on the creation of topological disorder due to the incorporation of a large concentration of 3 -fold coordinated boron atoms into the silicon network.

\section{Acknowledgements}

This work was supported by FAPESP and CNPq.

\section{References}

[1] W. E. Spear and P. G. Le Comber Solid State Communication 17, 1193 (1975).

[2] N. F. Mott, Adv. Phys. 16, 49 (1967).

[3] R. A. Street, Phys. Rev. Lett. 49, 1187 (1982). 
[4] M. Stutzmann, K. D. Biegelsen, and R. A. Street, Phys. Rev. B 35, 5666 (1987).

[5] I. Chambouleyron and D. Comedi, J. of NonCrystalline Sol. 411-417, 227 (1998).

[6] M. Vollmer, J. D. Meyer, R. W. Michelmann, and K. Bethge, Nuclear Instruments and Methods in Physics Research B 117, 21 (1996).

[7] H. Shanks, C. J. Fang, L. Ley, M. Cardona, J. F. De- mond, and S. Kalbitzer, Phys. Status Solidi B 100, 43 (1980).

[8] R. Swanepoel, Journal of Physics E 16, 1214 (1983).

[9] W. E. Spear and P. G. Le Comber, Solid St. Commun. 17, 1193 (1976).

[10] S. Kalbitzer, G. Müller, P. G. Le Comber, and W. E. Spear, Phil. Mag. B 41 (4), 439 (1980).

[11] M. Stutzmann, Phil. Mag. B 60, 531 (1989). 